\title{
A Combination of Augmented Reality and Google Earth's facilities for urban planning in idea stage
}

\author{
Viet Toan Phan \\ Ph.D candidate \\ School of Architecture\& Civil Engineering \\ Kyungpook National University, \\ Republic of Korea
}

\author{
Seung Yeon Choo \\ Assistant Professor \\ School of Architecture\& Civil Engineering \\ Kyungpook National University, \\ Republic of Korea
}

\begin{abstract}
This article presents a study on Augmented Reality (AR) development as a new performance approach for collaborative space of urban design work. Here, the participants- e.g. architects, structure engineers, equipment vendors, etc- can view an urban design process through AR scenes by Head-Mounted Display (HMD) glasses. Nowadays, the AR technology has been supported as a great graphical computing technology which promises to offer several advantages of applying for the digital architecture and construction fields. The AR effects enable users to examine $3 \mathrm{D}$ project models integrated into real environment in an intuitive way and real-time. Therefore, this research focuses on an establishment a kind of smart AR discussion space- the Augmented Reality Conference Desk (named as ARCdesk for short). As the result, the participants can examine a planning process of urban design work in 3D/4D multi-phases by intuitive ways. In particular, all participants also can examine the digital urban mock-up from free views and they also can interact with whole models (or each part) during architectural discussion. As the novelty of research, the participants were suggested to manage and create the input digital urban models basing on use a range of available Google Earth facilities which are usability for architectural design, such as the Google SketchUp, Google Building Marker and Google 3D Warehouse. This example also involves the conceptual combination of $\mathrm{AR}$ and $4 \mathrm{D}$ construction simulation within indoor condition in order to simulate the phases such urban growing up. The implementation bases on the main technical theme: the imagery vision- based AR tracking and the Tangible Augmented Reality.
\end{abstract}

\section{General Terms}

Applications of computer science in modeling, visualization and multimedia, graphics and imaging, computer vision, humancomputer interaction, et. al.

\section{Keywords}

Augmented Reality; vision tracking; collaborative AR; tangible AR; urban design.

\section{INTRODUCTION}

Nowadays, the Augmented Reality technology obtains many robust developments and it is employing widely in several engineering domains that related to a Graphic User Interface (GUI) theme. Come along with the specialties of architecture domain, the AR technology enables us to realize the early several useful and interest applications. In fact, these were a lot of researches for the new ideas to be laid-out which were considered as integrations of $\mathrm{AR}$ and architecture field. For example, the Luminous Table (H. Ishii, et al, 2002) [14] and the Authur system (T. B. Moeslund, et al, 2004) [15] were known as the previous examples of architectural collaborative which were defined as the fusion conceptualities of the virtual-real working phases. Yet, in this example, we propose another combination manner of the AR assistance for collaborative urban planning discussion within indoor environment. As we known, in the planning stage, the urban design and site planning work are the inherent activities gathering various the architects, civil engineers and construction specialists to work together in discussions and presentations. Here, the traditional drawing papers and physical 3D models would be replaced by the virtual models generated from the AR system. These series of virtual urban models can be overlaid on city map graphics projected on screen desk surface in order to establish the Augmented Reality Conference Desk system, which would be named ARCdesk in this work. Therefore, the ARCdesk system outcome promises to be new collaborative working equipment which enables architects to do their actions in work conveniently in the AR environment, such as to observe and interact with urban planning sequences in real-time. In the implementation, all participants would stand around the luminous surface of ARCdesk and make an urban discussing and planning together. They wear a HeadMounted Display glasses (HMD) and observe urban models from free views in conference room, then they interact with the virtual models through Tangible Interface to follow the plan scenario as the professional natural activities. These operating processes are real stranger than any previous systems because its system bases on the development and management method of the available urban materials. The Google Earth and its facilities, e.g. the Google SketchUp, Google Warehouse and Google Building Marker (these three applications will be call as Google Earth's facilities from now) were well-known as the available virtual full information globe and the auxiliary online edit-tools. In particular, the function combining of many Google Earth facilities was building-up and developing to support to model management and planning urban process. The huge information storage of Google facilities could be employed on ARCdesk in order to assist users in building-up and managing the urban information (2D/3D/4D) promptly. This research also keeps an interest in examining of AR tracking techniques- based on fiducial marker (due to 2 types of markers: graphical and physical marker). This technique can support to user the interaction capabilities with virtual urban models under the Tangible User Interface (TUI). The ARCdesk system promises to 
be a seamlessly integration of the CAAD on desktop and AR presenting environment for both architecture design and urban planning process.

\section{OVERVIEW OF AUGMENTED REALITY TECHNOLOGY}

\subsection{Augmented Reality technology}

Augmented Reality (AR) is a new exciting technology that involves the overlay of computer graphics on the real world. As a result, the users can see real world augmented with virtual objects through desktop screen or the AR specialized display equipments, and they can also interact with these digital objects in real-time. In conceptual, the AR is also grouped under the umbrella term Mixed Reality (MR), which refers to a multi-axis spectrum of areas that cover Virtual Reality (VR), Augmented Reality (AR), tele-presence, and other related technology [1] (Figure 1).

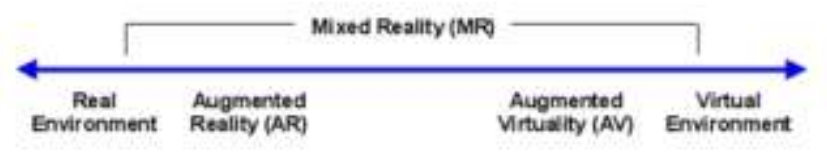

Figure 1. Paul Milgram's Reality- Virtual continuum.

Augmented Reality systems combine digital information and real world in the way that user can experiences them as one. A particular important of $\mathrm{AR}$ is locating virtual objects in the right place and position, which makes the Tracking System to be one of the most important components of an AR system. Essentially, an AR system must be able to follow the user's point of view dynamically and keep virtual objects aligned with real world objects. The basic needed components of an AR system are a display, camera, and processing computer with application software, and plus various kinds of portable device can be used, for example HMDs, camera phones, PDAs, electrical compass, lap-tops.

Although Augmented Reality has only been studied for over one decade, the growth and progress in the past few years have been remarkable. In particular, the AR applications have been using for digital life increasingly and also contributing to expand a range of Graphic User Interface (GUI) applications to many fields. As such, AR technology has many possible applications across a wide range of fields, including entertainment, education, architectural design, medicine, military training, engineering and manufacturing, etc.

It is also expected that other potential areas of AR application will be still appear with the disseminations of this technology in the next stages. During the early stages, the main focus of AR development was related to hardware technology rather than usability. However, the rapid development of mobile PC (handheld device) with better processing capacities and longlasting batteries has raised the issue of lightweight mobile AR systems. Thus, mobile AR devices also are now one of the most promising emerging technologies. However, the proposed system will be suggested to appeal to broader range of users basing on the use of regular PC and HMD. Thereby, in the study case, the aim of this AR system is to become an easy setting-up by conceptuality for architects. The valuable results of an imagerybased AR optical tracking method would help us to develop the achievements of the TUI realm in this work, and then it would be employed for the implementation absolutely.

\subsection{Related works}

Recently, the AR technology is being considered as a new method of design approachs for architecture. A lot of AR experimentations and researches are directed toward the architectural design process as the strong tools. As we known, one previous example of the collaborative space for architectural design is the Luminous Table project (H. Ishii, et al, 2002) [15] that also provided users with a simulated view of entire urban planning stage around the table (Figure 2-Left). Actually, this application proceeds from the result of a combination between 3 issues: the physical wireframe model, digital model and traditional drawing papers. It is merely the effects of graphical projection integrated with a bit of Augmented Reality concept. In other example, one previous project tend to the application of collaborative AR round table for architecture and urban design, it is Authur system (T. B. Moeslund, et al, 2004) [14]. The interaction effects of the Authur system were based on real world items (placeholder objects- PHO and a wand) and hand gestures. A PHO is a real object that acts as a placeholder for virtual object. It can be moved only on the table top, i.e. contains 3 degrees of freedom (DoF).This system purely focused on an establishment of AR Tangible Using Interface (TUI) content. There, the research expectations are the interactive capabilities of user with virtual building within AR scene. Actually, those previous researches also act as the source of new ideas or study background for other following researches around urban design work indoor.

Hence, in this example, we desire to examine and illustrate a kind of application that is bridging the gap between urban design works on desktop (known as CAAD) and representation process within AR environment. The concepts of Augmented Reality Tangible Interface would be combined with a imagery- based AR optical tracking method to be deeply exploited so that illustrate a new way operating of the ARCdesk. Then, it is examined to become the professional architectural equipment for architects. Therefore, the creation for input data of proposed system would be used from around the architectural materials, i.e. CAAD software or web-based facilities. The proposed system would be expected to be popular working tool for architects, engineers, designers, etc. or even if using for the users who have not enough experiences in computer science knowledge.

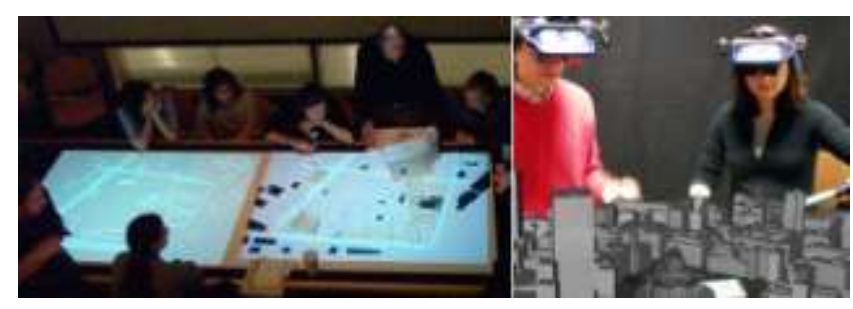

Figure 2. Left: Architectural students discussing at Luminous Table. Right: Viewing and manipulating virtual building in Authur round table meeting. 


\subsection{Imagery- based Augmented Reality optical tracking}

The precise registration of real and virtual objects in the real space is a significant research problem of AR field. Doctor Hirokazu Kato (1999) provided a good review of the issues related to the $\mathrm{AR}$ tracking and registration, and there are numbers of possible tracking approaches that can be used to develop Tangible AR tracking. Actually, this study applied a computer- vision based method in which the virtual models can be fixed in right space relative to one or several neighbor tracking markers. As usual, these AR markers are known as simple black squares with a unique pattern inside them. The approach of AR tracking based on fiducial marker is illustrated in figure 3. After threshold input image, square markers are extracted and identified, and then the pose and position of the markers are estimated from the coordinates of 4 vertices. Finally, virtual images are drawn on the input marker accurately.

The study was chosen by using ARToolKit library. The ARToolKit is free well-known AR application which is programmed by using C\# language. In general, ARToolKit can overlay virtual imagery on the real world objects conveniently. In details, ARToolKit captures the image patterns from the camera input, analyzes the image and searches for predefined patterns. If any image pattern is found then the toolkit overlays the corresponding virtual object over the real object. Corresponding to the system research, a dozen of patterns for fiducial markers were produced by ARToolKit library, and then they would be grouped under various functions of ARCdesk system.

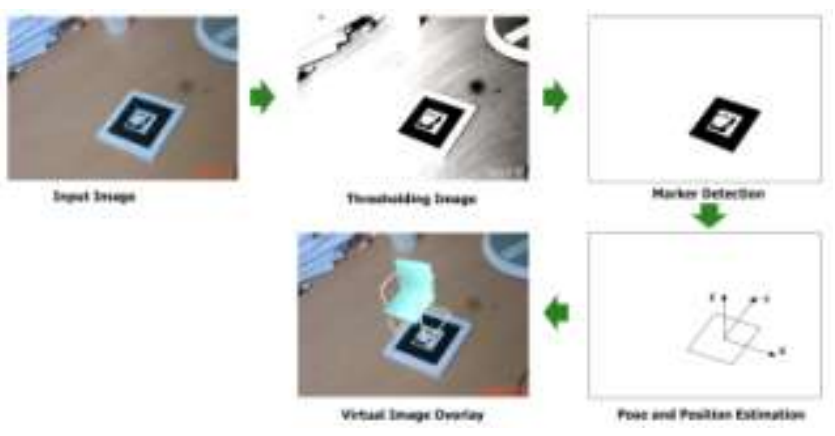

Figure 3. Figure marker- based AR tracking process.

In tracking process (Figure 3), a size- known square marker is used as a base of the marker coordinates in which the virtual objects are represented. ARToolKit then use the known square size and pattern orientation to calculate the position of the real video camera related to the physical marker. Then the goal is to identify the transformation matrix $(3 \times 4$ matrix) from the marker coordinates to the video camera real world coordinates. This matrix is then used to set the position of the virtual camera coordinates. This matrix consists of the rotation values and translation values, which was explained in a previous essential Augmented Reality study of H. Kato (2008). There, these values are found by detecting the markers in the camera image plane and using perspective transformation matrix. Since the virtual and real camera coordinates are the same, the computer graphics that are drawn precisely overlay the real marker. The OpenGL AP is used for setting the virtual camera coordinates and drawing the virtual camera.
In this work, the executing of the tracking method is assumed by video-see-through AR glasses united video camera, where the video frames shown on user's display is the same frame used to track user's viewpoint, allow the virtual model to appear as an exact overlay in real environment. In general, the AR tracking patterns were used following two forms of appearance manner: the physical marker for user's direct interaction and graphical marker projected on desk surface in real-time.

\subsection{Occlusion marker- based Tangible Augmented Reality}

This work applies an interactive object-centered view to $2 \mathrm{D}$ interaction effect, which is easy to apply to Tangible AR environments where natural interaction methods are vital. In the real world, users are able to use a variety of objects or even their bare fingers as a pointer. In addition, for some situations with multiple participants or bi-manual interactions, the interaction can even involve multiple pointers.

Detecting pointer over an interaction object can be achieved in numerous ways, where detection the occlusions of the tracking marker is a passive way to detect pointing actions. The occlusion of an AR marker can be easily utilized as an interaction method in Tangible AR environments in which a camera is already available for providing real-world views to the user and tracking the objects of interest with passive formal markers.

For occlusion detection, the predefined tracking markers are widely used for tracking real objects in Tangible AR environments. Two ways of occlusion marker- based Tangible AR methods are explored in this work. First of all, vision camera-based tracking systems usually require multiple markers for tracking one object. A number of markers are assigned to a single object in a pre-configured spatial relation ship. In this way the object can be tracked successfully even if some markers in marker set are not visible. In addition, because the spatial relationships of all the markers are known, the poses of markers that are not visible can be estimated using the markers that are recognized. Plus, the pose of each marker set of case are predefined when one unit marker are occluded by user. Therefore, several tracking marker case can be pre-definded from each full marker set.

A simple way to guarantee that the marker is within the view volume is to check the visibility of its neighboring markers, referred to as boundary markers (B symbol), while a marker being checked for occlusion is referred to an interaction marker (I symbol).

To guarantee that an interaction marker is within the view volume, the boundary markers must be placed carefully. The convex hull of the boundary markers must include the interaction marker. For instance, for a single interaction marker, at least 2 boundary markers are needed surrounding the interaction marker (Figure 4- Left). By checking whether these boundary markers are visible, the interaction marker can be guaranteed to be within the view volume, making its occluded value if it is not detected full. 


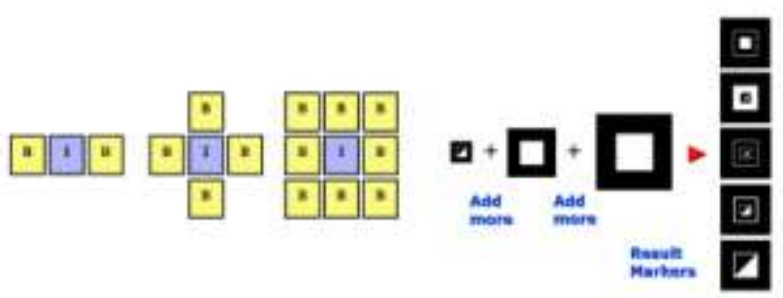

Figure 4. Left: Boundary markers around interaction markers. Right: Multi- Class marker prototype.

In the second type of occlusion marker, this study also contributes the interaction method based on a Multi-Class marker set (Figure 4-Right), which is developed basing on an imagery identifiable capability of the computer- vision tracking process. In fact, a Multi-Class marker combines the functions of several unit marker values. The operating activities of a MultiClass marker set are through an assembling manipulation each unit physical markers (or occluding unit marker shapes). Theoretically, there are several unit marker values to be created within one Multi-Class marker. Yet, in this example, one MultiClass marker consist six unit marker values is produced for initial AR simulation of urban models on the ARCdesk.

\section{GOOGLE EARTH'S FACILITIES FOR SUPPORTING TO URBAN PLANNING}

\subsection{Facility tools for Google Earth}

Here, we examine the Google Earth's facilities which are available tools supported to 3D Google Earth domain: Google SketchUp, Google Building Marker and Google Warehouse.

Following advances of the Google Earth program, these facility tools have been aiding and improving incessantly. In architecture realm, these applications strongly demonstrated the capabilities which can support to creating-up process of virtual cities world for broad engineering applications. Basing on the Google Earth, Google user now can promote the discovering cities, countries, 3D model of buildings, satellite and aerial imageries, terrain, and much more in any areas.

In fact, the Google Earth is virtual globe, rich of map and geographic information program that was originally call Earth Viewer 3D. It maps the Earth by the superimposition of the images which obtained from satellite imagery, aerial photography and GIS 3D globe.

Nowadays, the available Google Earth's facilities are released by Google Inc made information repository of Google Earth to be rich and huge. The specialty is aimed at 3D models information of the buildings in cities. Hence, we propose the building- up methods to modeling of urban mock-ups based on the Google Earth's facilities and available building models repository which is involved the managing and new creating- up capabilities. The figure 5 shown the schema could be explanation about reciprocal relationship of inner Google Earth's facilities.

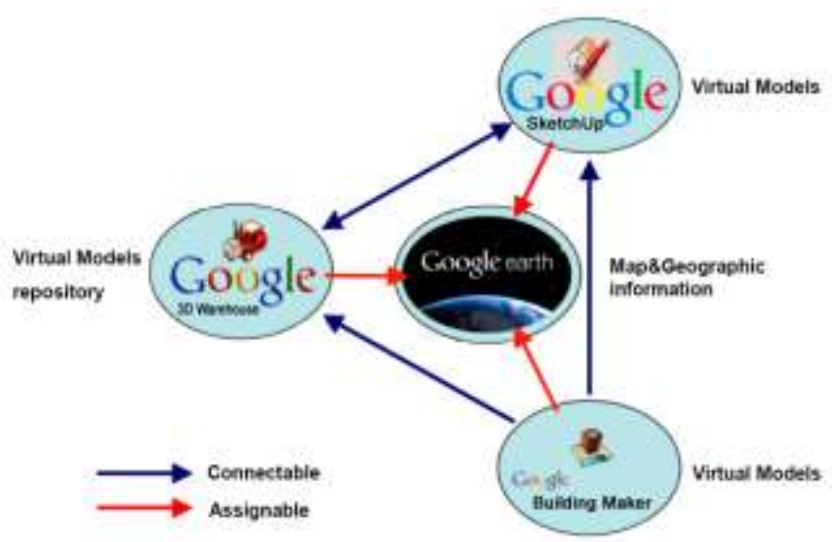

Figure 5. Reciprocal relation of inner Google Earth's facilities.

This schema is able to suggest user exploiting the available relationship of Google Earth's facilities, so that gathering the great number of urban object materials which is aimed supporting to Augmented Reality functions of ARCdesk. The explanation of exploiting Google Earth's facilities is expressed in follow section.

\subsection{Google Earth's facilities- based the creation and storage 3D building models}

The Google Earth's facilities, involved the Google SketchUp, Google Building Marker, Google Warehouse, were known as the effective tools can help user interact with virtual globe of Google Earth. In addition, these tools also can assist the architects or engineers to create and store numerous 3D models of buildings or the bits in the world. This is the core issue in order to create the link from urban design to the Augmented Reality applications. Almost all model files will be generated for the AR application through Google SketchUp platform. These model files are converted to common 3D computer graphic files such as VRML or OBJ extensions.

Actually, the Google Building Marker still is a new facility which was released by the Google Inc at the end of 2009 years. This specialized online tool is improving and expanding the applied limitations to many cities in over the world. In present, the users can use it to make buildings in any of about 50 cities. Google Inc still keeps steps adding more cities in near future.

In this research, the chosen area for creating urban planning mock- up is the limited zone in the Seoul (Korea) where is an area around the Namdaemun Gate. The creating buildings process through Google Earth's facilities will be summarized in below sections.

- Google SketchUp is the 3D modeling software that allow user to create anything freely, including photorealistic models for Google Earth. The SketchUp assumes to produce the extra urban models or other components which can not be found from 3D Warehouse. Simultaneously, the Google SketchUp also has capabilities to convert all instant urban models which were generated from CAD software. 


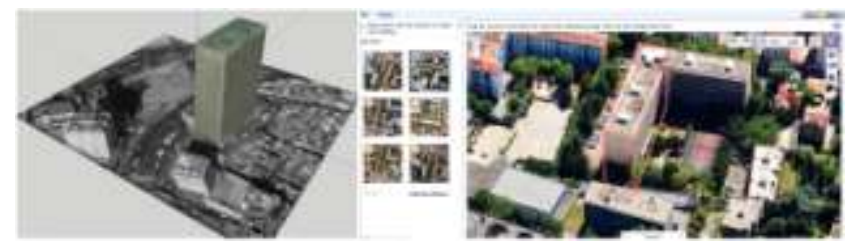

Figure 6. Left: Model of the Lotte Insurance building created in SketchUp. Right: Building model is created online in Google Earth by Google Building Marker.

- Google Building Marker is an online 3D modeling tool for adding the 3D buildings to Google Earth promptly. It's simple and interest to use, and an easy to get on the 3D map. Thus, millions of people will be able to see the created models in Google Earth. The models created in Building Marker can be easily modified simultaneously through Google SketchUp software. However, the Building Marker is not supported to replace the SketchUp. SketchUp is a general- purpose tool that people use to model everything under the sun, the Building Marker just is instant online tool to create buildings conveniently.

- In this work, Google Warehouse would be contributed as useful one to exploit urban buildings. Google Warehouse is free, online repository where user can find, share, store, and collaborate on 3D models. It is an easiest way to share private model of a place with the millions of Google Earth users by uploading to Google Warehouse. Serving for experiment, some main building models were converted from Google Warehouse repository, e.g. Namdaemun Gate, Lotte Insurance building, Shinhan Bank Headquater building, etc.

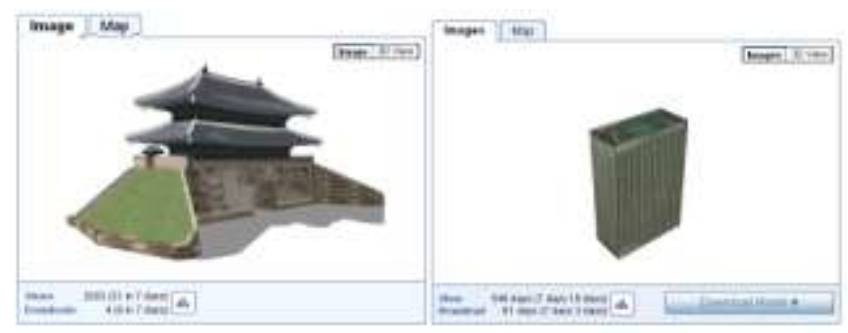

Figure 7. Namdaemun Gate\& Lotte Insurance building models in Google Warehouse.

In general, the large of building models located in examined area were created and found through using right Google Earth's facilities. As a research's purpose, these models are gathered, and then will be used to simulate as the virtual 3D objects within the proposal AR system.

\section{ESTABLISHMENT OF URBAN PLANNING CONFERENCE THROUGH ARCDESK SYSTEM}

\subsection{The combination of Google Earth's facilities and $A R$ technique}

As above mention, the Google Earth's facilities are the huge repository of geographic and 3D buildings information in the world. Plus, these facilities also involve flexible capabilities of creating more 3D model of buildings or other bits. The Google Earth's applications hold strong capabilities to generate out two issues: 3D model data of buildings and a range of map images. This study aims to examine those two issues and layout the creation of real-virtual mock-up (ARCdesk). The geometry data of 3D models generated from Google Earth applications will be produced as virtual graphics within AR processing of ARCdesk. Those digital objects were stored readily in database of proposed AR system. As shown in section 2, the AR environment will be focused to setting-up basing on two issues which are processed from ARToolKit: the supporting 3D objects and the image- based tracking method. Hence, whole of 3D building models would be generated according to the urban study case, and then to be delivered to $\mathrm{AR}$ applications through digital 3D computer graphic files (extension file as VRML or OBJ forms). The building models related study case are defined and assigned to the AR fiducial markers previously. In implementing process, the interaction of user with 3D models will be based on the transformation of the AR marker images. Therefore, the participants will work and interact with the building models on the map through physical manipulations with AR marker.

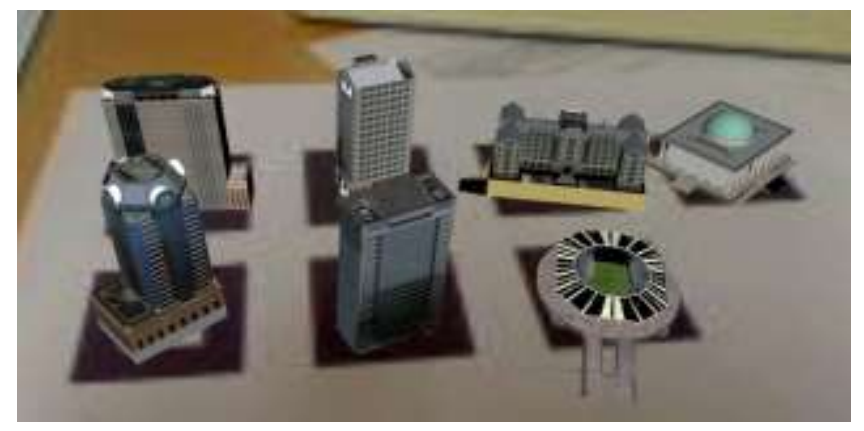

Figure 8. The building models generated from Google Earth's facilities are superimposed in AR scene.

\subsection{System working diagram}

For the implementation, two separate models were developed: one for creating and managing 3D geometries, and the other for AR displaying functions, as show in the figure 9 by system diagram.

First of all, the Google Earth's applications generate building information from inner themselves or outside sources, and then link it to database of system. After loading a 3D geometry of buildings, the position and direction of the user's views are calculated based on marker tracking method. Simultaneously, the location and direction- bases on geometry data- are transformed through using transformation matrices in order to produce $3 \mathrm{D}$ graphic of buildings that to be aligned beside other physical objects in the real view. As such, an AR tracking process is important element of proposal AR systems.

The 3D building geometries are stored in graphical database which was generated by the combination of Google Earth's applications, while the OpenGL program assumes to render the final augmented graphics onto AR display (HMD). The ARToolkit library software is using as the AR processing in this example. The effects from AR marker development can support to user the interaction capabilities with virtual models in operation. 


\subsection{Hardware}

This study aimed to create the ARCdesk as working equipment for architects and engineers. The ARCdesk is a virtual- real technical desk using for an architecture discussion and presentation. The most function of this equipment is the use for urban study process. Simultaneously, the virtual models of buildings and other bits of the cities can be superimposed on correct position of the graphic maps, which are projected spread on horizontal flat surface of the ARCdesk. Therefore, the participants easy to visualize the virtual building models integrated on projected map, and then they also can interact with them through manipulating with physical markers (Tangible AR). At the same time, the other audiences within the conference room can watch the discussion through keeping follow on projector screen in real- time. The one video camera is connected to AR application also is reserved for that processing, and then it would be posited beside of the ARCdesk. The working system in the figure 12 will list the principal equipments that composed the proposal ARCdesk system.

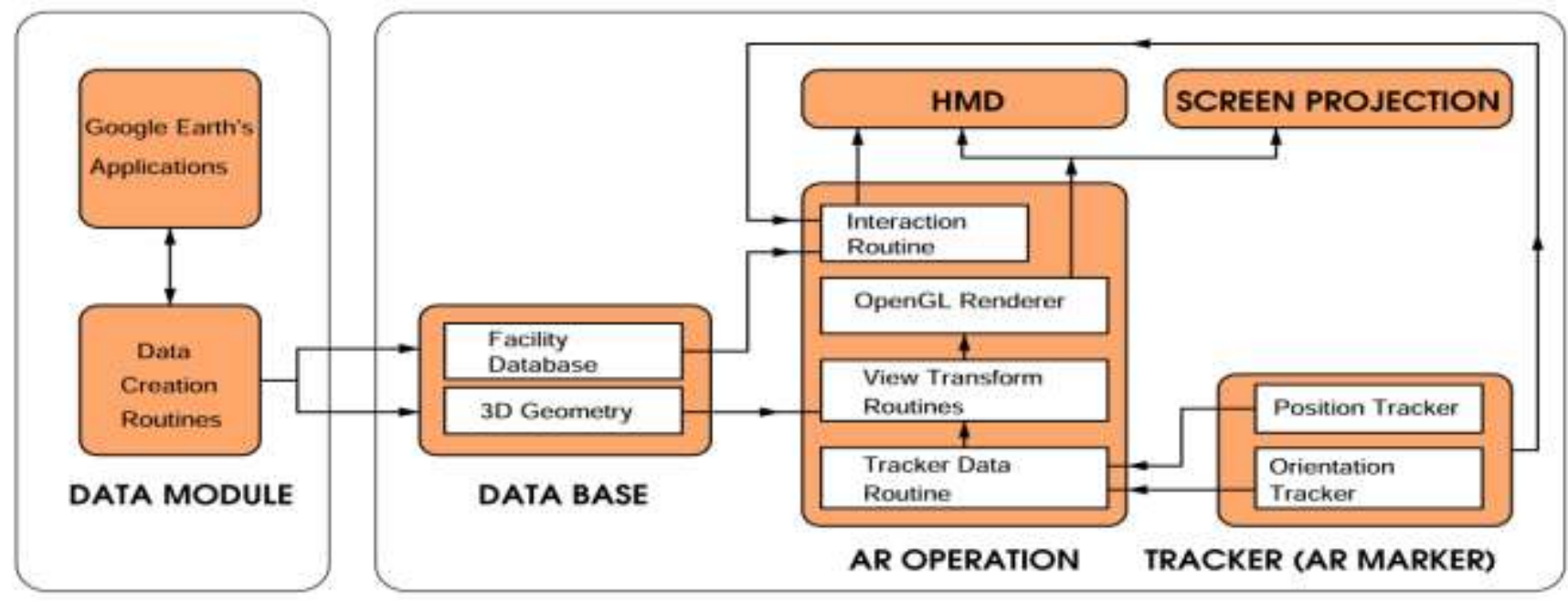

DATA CREATION

DISPLAY MODULE

Figure 9. Augmented Reality System Diagram.

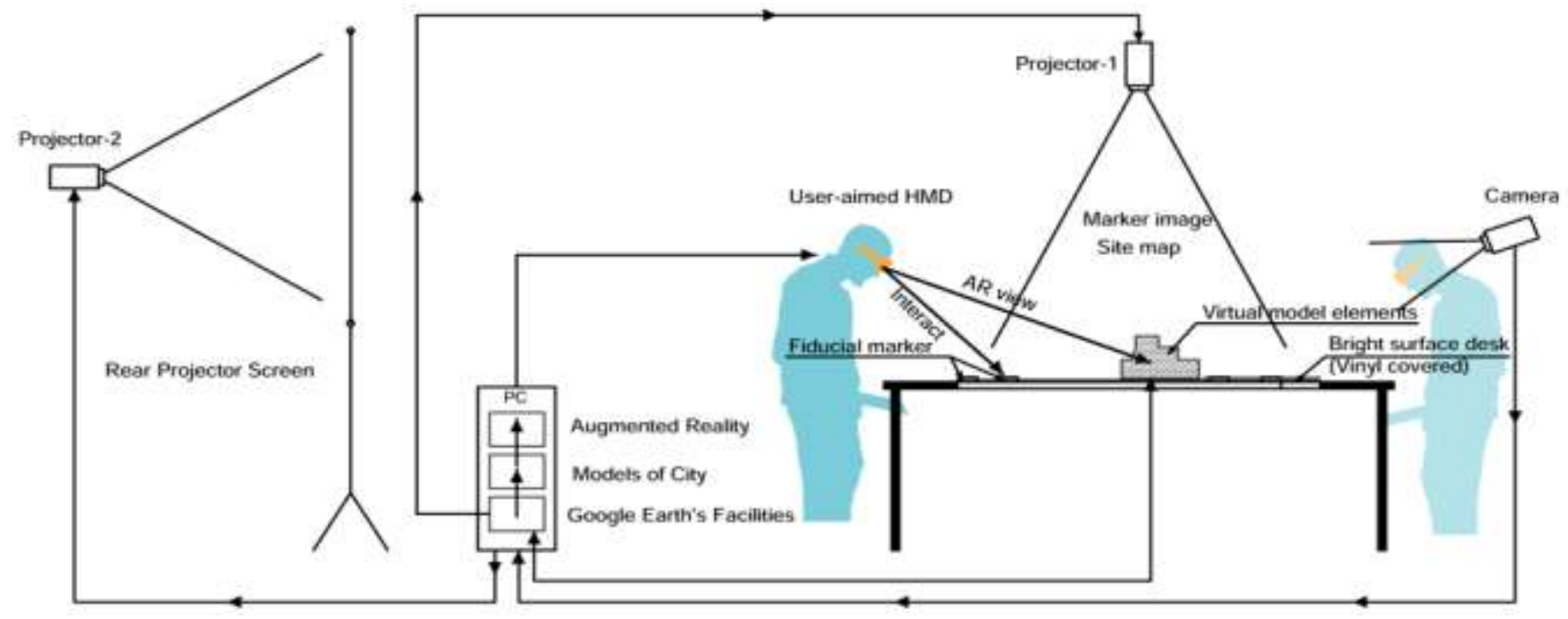

Figure 10. Working System Scheme.

\section{- Processing Computer}

In the implementation, the prototype AR system operated based on desktop PC installed Windows XP operating systems running on an Intel(R) Core(TM) Quad CPU Q6600 with 2GB of RAM.
Plus, a HandyCam camera is used to capture video streams of AR urban mock- up due to the experiment.

- Head Mounted Display (HMD) for AR 
The HMD mainly consists of two parts: a video input device and displays. Almost any kind of video input device can be used as the video stream captured device (i.e. integrated video camera). Yet, since the main purpose of this study is to provide a complete AR scene for users working under free walk condition, the devices need be compactable. Therefore a video- see through HMD- VR Pro AR $800 \times 600$ - is used to generate the virtual objects on the real scene. While user is still enabled to see real scenes around of physical design space, the virtual graphics of urban models are overlaid on eye- monitors. The figure 11 shows an example of how components of the Augmented Reality system working together to produce the final results on video eyemonitors.

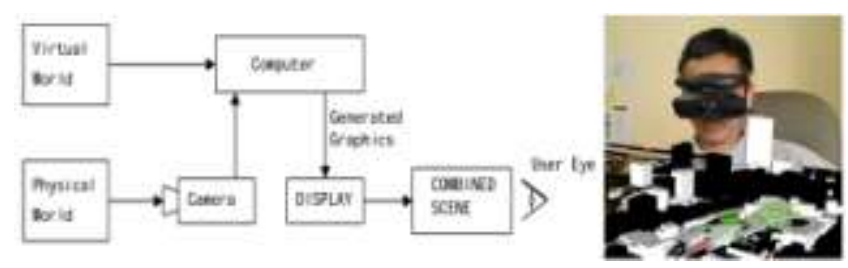

Figure 11. Schematic illustration of video overlaid- based Augmented Reality\& VR Pro AR 800x600 HMD.

The determination of user's exact position and orientation parameters, so- called pose, is based on a video tracking algorithm that analyzes the user's current view of special features, which is why the camera has to be compacted to the display unit in order to keep capturing the field-of-view and transferring the image sequence to the AR processing module. The more image the system can process, the more accurate the pose that can be extracted.

\section{- Marker images}

As mention in section 2.3, the ARToolKit library assumes developing the AR applications in this research. The fiducial markers generated from ARToolKit library will be assigned to predefined virtual objects. Total 9 marker patterns were created to be used at two forms concurrently for implementation: the physical markers (foam card fixed image paper) and the projected markers integrated into city maps on the desk surface. In particular, some other marker sets also are created to use for occluded marker- based Tangible AR interaction.

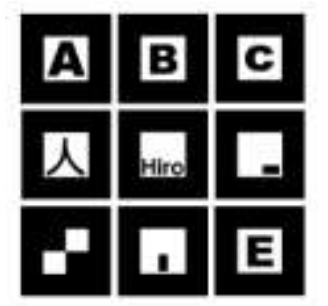

Figure 12. AR fiducial markers created ARToolKit

- Projector and other devices

The ARCdesk is proposed to use portable projector with high resolution (1920x1080 pixels) and contrast ratio (5000: 1). Plus, the other needs output devices, such as screen table and rear projector screen, also are employed in order to present audiences entire of professional activities on ARCdesk.

\subsection{Implementation and discussions}

This section will interpret the main part of experiment results, and the reflection of those results within context of the framework which can be established from perspective of urban planning and architectural discussions. The scenario of research proposed the using process of ARCdesk system. That means the architects, students and other audiences are able to use the ARCdesk as assisted equipment for urban planning discussion of the limited city area- the pre-defined area around Namdaemun Gate in Seoul. In the operation of the proposal system, the foamcards sticks paper markers are toolkits that enabled user to interact with building models, which are real reserved for creating a virtual mock- up of the urban planning according to proposal designer's ideas. In addition, as above mention, the markers overlaid on screen-desk are pre-posited on the map to be relative with working scenario (Figure 13-Left). These projected markers are controlled by computer processing during working time. The user's interaction with virtual model is operated by user touch activities on the markers in order to hide parts of marker/or marker set, as mentioned in section 2.4.

For working on an ARCdesk, each of presenters is equipped HMD glasses and they are able to catch and hide virtual models overlaid on ARCdesk in real-time. Subsequently, the participants can move virtual building models or other bits through moving actions the physical markers on virtual projected maps of ARCdesk. In other stage, the participant proposes out the prototype of an urban module which has been planned before and posits it on the city map for comparison (Figure 13- Right). This work is dealt through the Tangible AR- based marker interaction. This process also would be sample if user wants to order which urban components overlaid on (buildings, parks, infrastructure systems, etc). Meanwhile, the other audiences can keep an watch discussion process through the AR sub-system which can provide an entire of the urban mock-up onto projector screen.

In contrast to traditional urban study, the physical models made from soft material, this example enables user examines an urban mock-up in Immersive Virtual Environment (IVE). In present, along with most stages of urban design works are built-up and controlled by computer which was known as the CAAD fields, this study also promise to be the useful theme for following development. In the early stages of design process, a design information data is passed into 3D object database. Especially, numerous of available 3D models data from web are employed for creating-up the intuitive working environment for urban designers. Here, the miniature physical models are replaced completely by the AR virtual models. During design work in AR environment, whole senses and effects in the comparison with working by physical models could be unchanged. Some architects and students in campus took an experience with the ARCdesk, and then they commented that the ARCdesk would be able gained many advance effects and interested as such they have not known before.

\section{CONSLUSIONS}

This study one more explored the potentialities of AR application for an architectural design. The results suggested a new working environment for architects- IVE environment- through the Augmented Reality Conference Desk (ARCdesk) equipment. As 
mention in the body paper, the proposal AR system can support architects to create up an urban virtual mock-up to be replaced traditional physical models in future work. Hence, they can manipulate design works in a seamless virtual-real environment. The ARCdesk also enable user to interact and observe urban models with free moving through HMD glasses. By the

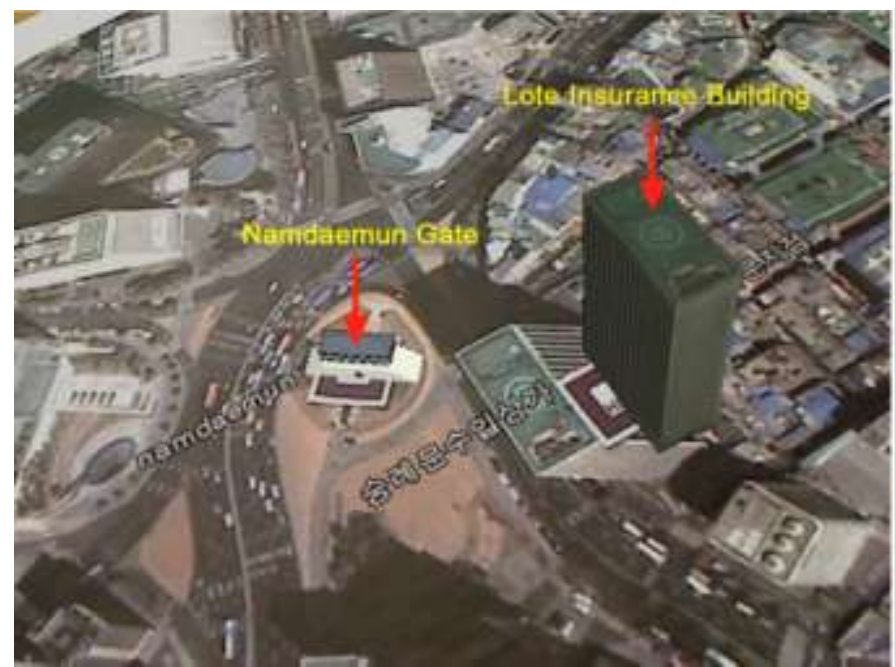

demonstrated issues above, the ARCdesk system is an actual virtual-real collaborative space for architects and students using in architectural education. Here, all participants can search and share a range of architectural ideas easily, and then they are able to establish new working manners.

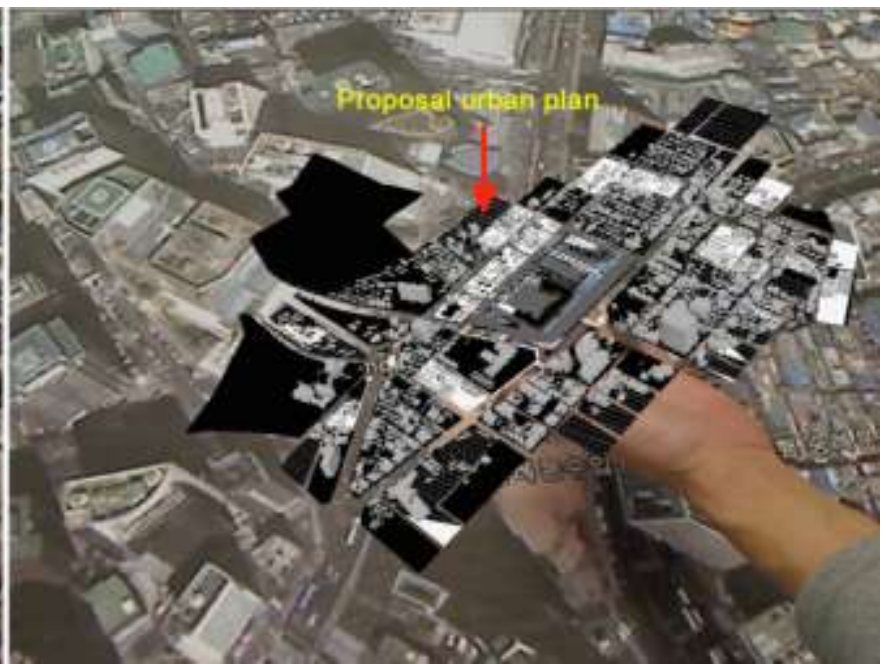

Figure 13. Left: Building models placed on map screen. Right: User manipulating with urban plan of examined area.

Nowadays, the AR techniques is developed and get precious results continuously as dozen of AR tracking methods. Thus, this study presented the initial results of the combination between $\mathrm{AR}$ and architecture fields, and it aimed to demonstrate about the proposal working manner. Next times, we will continue to examine the technical advances of using AR effects embedded ARCdesk structure aimed to develop and extend a broader range of usability.

\section{ACKNOWLEDGMENTS}

This research was supported by Basic Science Research Program through the National Research Foundation of Korea (NRF) funded by the Ministry of Education, Science and Technology (2010-0015999).

\section{REFERENCES}

[1] Azuma, R. 1997. Presence: Teleoperators and Virtual environment. Citeseer, USA. Vol. 6, Issue 4, 355-385.

[2] Billinghurst, M., Kato, H. and Poupyrev, I. 2008. Tangible Augmented Reality. In Proceedings of ACM SIGGRAPH ASIA, Singapore.

[3] Billinghurst, M., Poupyrev, I., Kato, H., and May, R. 2000. Mixing Realities in Shared Space: An Augmented Reality interface for Collaborative computing. In Proceedings of ICME, IEEE, USA. 1641-1644.

[4] Dias, J. M. S., Santos, P. and Nande, P. 2003. In Your hand Computing: Tangible Interfaces for Mixed Reality. In Proceedings of $2^{\text {nd }}$ IEEE International Augmented Reality ToolKit Workshop, Waseda University, Tokyo, Japan.
[5] Kensek, K., Noble, D., Schiler, M. and Tripathi, A. 2000. Augmented Reality: An application for architecture. In Proceedings of $8^{\text {th }}$ International Conference on Computing in Civil and Building Engineering, ASCE, Stanford, CA. 294-301.

[6] Lee, G. A., Nelles, C., Billinghurst, M. and Kim, G. Immersive authoring of Tangible Augmented Reality application. In Proceedings of the 3nd IEEE/ACM International Symposium on Mixed and Augmented Reality. 172-181.

[7] Milgram, P., Takemura, H., Utsumi, A. and Kishimo, F. 1994. Augmented Reality: A class of displays on the realityvirtual continuum. In Proceedings of the Telemanipulator and Telepresence technologies, SPIE, Bellingham, Wash. Vol. 2351, 282-294.

[8] Pasman, W. and Woodward, C. 2003. Implementation of an Augmented Reality system on a PDA In Proceedings of $2^{\text {nd }}$ IEEE/ACM International Symposium on Mixed and Augmented Reality. 276.

[9] Sherman, W. and Craig, A. 2002. Understanding Virtual Reality: Interface Application and Design. MorganKaufmann Publisher.

[10] Woodward, C., Lahti, J., Rökkö, J., Honkamaa, P., Jäppinen, J., et al. 2007. Virtual and Augmented Reality in the digital building projects. International Journal of Design Science and Technology. Vol. 114, No.1, 23-40.

[11] Vallino, J. R. 1998. Interactive Augmented Reality. Dortor of Philosophy Thesis. University of Rochester New York.

[12] Siltanen, S. and Woodward, C. 2006. Augmented interiors with digital camera images. In Proceedings of Seventh 
Australia User Interface Conference, CRPIT 50, Australia. 33-36.

[13] Chen, R. and Wang, X. 2008. An Empirical Study on Tangible Augmented Reality Learning Space for Design Skill Transfer. Tsinghua Science \& Technology. Vol. 13, No. 1, 13-18.

[14] Broll, W., Lindt, I., Ohlenburg, J., et al. 2004. AUTHUR: A Collaborative Augmented Environment for Architecture Design and Urban Planning. Journal of Virtual Reality and Broadcasting. Vol. 1, No. 1, 1-10.

[15] Ishii, H. and Joseph, E. B. 2002. Augmented Urban Planning Workbench: Overlaying Drawings, Physical Models and Digital Simulation. In Proceedings of IEEE/ACM International Symposium on Mixed and Augmented Reality (ISMAR), Darmstadt, Germany.

[16] Harmut, S. and Aurel, S. M. 2005. Digital and Tangible Snesation: An Augmented Reality Urban Design Studio. In
Proceedings of $10^{\text {th }}$ Conference on Computer- Aided Architectural Design Research in Asia, New Dehli, India.

[17] Buchmann, V., Violic, S., et al. 2004. FingARtips- Gestur Based Direct Manipulation in Augmented Reality. In Proceedings of $2^{\text {nd }}$ International Conference on Computer Graphics and interactive techniques, ACM Press, New York. 212-221.

[18] Wang, X. and Gu, N., et al. 2007. Perceptions of Augmented Reality in the Design Sector. In Proceedings of $7^{\text {th }}$ International Conference on Construction Applications of Virtual Reality (CONVR2007), Pennsylvania State University, USA. 132-138.

[19] Chen, C. H., Hsu, M. F., et al. 2009. Applying Augmented Reality to Visualize the History of Traditional Architecture in Taiwan. In Proceedings of $22^{\text {nd }}$ CIPA Symposium, Kyoto, Japan. 